\title{
BY WAY OF PREFACE
}

The invitation to participate in the collection Dialogues and to sketch in this framework our personal experiences as explorers naturally elicited from both of us a deeply sympathetic response.

In language and in the science of language the presence of an interlocutor is of fundamental importance: the mastery of language is a dialogue, and the development of language is also a dialogue. The account of the relations between individual and collective contributions to the science of language and of its artistic transformation gains in productivity when submitted to discussion. Linguists have noted the existence of tribes which are familiar only with dialogic speech and for which monologues are totally alien. More precisely, these are tribes whose only speech outside of dialogues consists of ready-made ritualistic monologues. In our usual cognitive speech as well, monologues, much more than dialogues, are subject to ritualization. The temporal and transient shape of both creative speech and meditations on verbal creativity lends itself much more easily to critical debate than to individual report. 
